\title{
Origin Of The Cosmic Ray Positrons Observed Near Earth- Meson Decay Or Dark Matter Decay?
}

\author{
Shlomo Dado ${ }^{1}$ and Arnon Dar ${ }^{1}$
}

\begin{abstract}
We show that the flux of the high energy cosmic ray positrons observed near Earth is that expected from the decay of mesons produced by the primary cosmic rays (CRs) in the local interstellar medium.

Subject headings: cosmic rays, dark matter
\end{abstract}

\section{Introduction}

A wide variety of evidence points to the existence of dark matter in the universe which cannot be seen directly, but which can be detected by its gravity. Precise measurements of the angular power spectrum of the cosmic microwave background radiation analyzed with the standard model of cosmology indicate that nearly $27 \%$ of the total mass-energy density of the universe is residing in this dark matter (Bennet et al. 2013; Ade et al. 2014) whose existence was first discovered in clusters of galaxies (Zwicky 1933,1937) and later in galaxies (Rubin 1970). The nature and origin of this dark matter (DM) are still unknown.

If the dark matter is made of weakly interacting massive particles (WIMPs) relics from the big bang, their annihilation cross section satisfies $\left\langle\sigma_{\mathrm{w}} \mathrm{v}>\approx 2 \times 10^{-26} \mathrm{~cm}^{3} / \mathrm{s}\right.$ (Jungman et al. 1996), which is typical of weakly interacting $\sim \mathrm{TeV}$-mass particles. Annihilation/decay of such particles can produce stable particles such as electrons, positrons, protons, antiprotons, neutrinos and gamma rays, whose energy cannot exceed the mass of the DM particles. Hence, in recent years, bumps with a sharp cutoff due to the decay and/or annihilation of WIMPs were looked for in high precision measurements of the energy spectrum of such cosmic ray (CR) particles. A bump around $\mathrm{E}=620 \mathrm{GeV}$ with a sharp cutoff around $\mathrm{E}=800$ $\mathrm{GeV}$ in the combined flux $\Phi_{\mathrm{e}^{ \pm}}$of $\mathrm{e}^{ \pm}$cosmic rays (CRs) was reported by the Advanced Thin Ionization Calorimeter (ATIC) balloon experiment (Chang et al. 2008). It was interpreted as a possible dark matter signal (e.g., Bergstrom et al. 2008; Cholis et al. 2009; Cirelli et al. 2009; Arkani-Hamed et al. 2009; Pohl 2009). However, such a peak was not confirmed

\footnotetext{
${ }^{1}$ Physics Department, Technion, Haifa 32000, Israel
} 
by measurements with the ground based Cherenkov Telescopes of High Energy Stereoscopic System (H.E.S.S.) (Aharonian et al. 2009), and later with more precise measurements with the Large Area Telescope (LAT) aboard the Fermi satellite (Ackermann et al. 2010) and with the Alpha Magnetic Spectrometer (AMS) on the International Space Station (Aguilar et al. 2014). But, at the same time, the energy spectrum of $\mathrm{e}^{ \pm}$CRs measured with H.E.S.S. (Aharonian et al. 2008) showed a sharp break near $1 \mathrm{TeV}$ whose origin is still not clear.

Shortly after the ATIC report (Chang et al. 2008), an unexpected increase of the CR positron fraction $\Phi\left(\mathrm{e}^{+}\right) /\left(\Phi\left(\mathrm{e}^{+}\right)+\Phi\left(\mathrm{e}^{-}\right)\right)$as a function of energy between $\sim 10 \mathrm{GeV}$ and $\sim 100$ $\mathrm{GeV}$, observed with the Payload for Antimatter Matter Exploration and Light-nuclei Astrophysics (PAMELA) satellite was reported (Adriani et al. 2009,2010,2013). Unlike the ATIC bump, the rise with energy of the positron fraction has been confirmed by measurements of the separate CR ray fluxes of $\mathrm{e}^{ \pm}$with Fermi-LAT (Ackermann et al. 2012), and with AMS (Aguilar et al. 2013,2014; Accardo et al. 2014) with much larger statistics and smaller systematic errors. In the high precision AMS data, this rise continues beyond $100 \mathrm{GeV}$ but with a decreasing rate that appears to level off around $275 \pm 32 \mathrm{GeV}$ (Accardo et al. 2014, Aguilar et al. 2014, Pohl 2014).

The measured flux of CR positrons and the increasing positron fraction were in stark contrast with those predicted for secondary production of positrons in the interstellar medium (ISM) by primary CRs from detailed calculations with the elaborate GALPROP code (e.g., Moskalenko and Strong 1998a,b). This sparked the publication of several alternative explanations to the origin of the excess of high energy $\mathrm{CR} \mathrm{e}^{+}$flux, such as dark matter (e.g., Bergstrom et al. 2008,2009,2013; Bergstrom 2009; for a recent review see, e.g., Ibarra et al. 2013 and references therein), positron emission from a few nearby pulsars and/or supernova remnants (e.g., Hooper et al. 2009; Shaviv et al. 2009), secondary production of positrons in collisions of primary CRs in/near their sources (Dado \& Dar 2010) such as the highly relativistic jets launched mainly by supernovae Type Ic and acreting stellar mass and massive black holes (e.g., Dar and De Rújula 2004, 2008 and references therein) and/or the spherical ejecta in supernova remnants (SNRs) (Blasi 2009; Stawarz et al. 2010). Other authors (e.g., Blum et al. 2013; Cowsik et al. 2014) maintained that secondary production of positrons in the ISM by the primary CRs can explain the observed flux of CR positrons although their calculations involved incorrect assumptions concerning the energy-loss of positrons in the ISM.

The reported isotropy of the flux of high energy CR positrons observed with AMS (Accardo et al. 2014), however, is in tension with the assumption that the observed flux of $\mathrm{e}^{+}$CRs was produced by a few relatively nearby pulsars (e.g., Linden and Profumo 2013) or supernova remnants. At the same time, the high precision data of AMS02 (Aguilar et 
al. 2014) on the combined $\mathrm{e}^{ \pm}$flux up to $1 \mathrm{TeV}$ and the separate $\mathrm{e}^{-}$and $\mathrm{e}^{+}$fluxes, and the positron fraction (Accardo et al. 2014, Aguilar et al. 2014) that appear to flatten above 200 $\mathrm{GeV}$, do not show yet any convincing evidence for a cutoff/sharp decline, which begins below $\sim 700 \mathrm{GeV}$ that could be associated with a decay/annihilation of dark matter, particles.

In this paper, we re-examine the possibility that the main origin of the high energy CR positrons observed near Earth is secondary production in hadronic collisions of the primary cosmic ray protons and nuclei in the ISM (Blum et al. 2013; Cowsik et al. 2014). We focus on the high energy $(\mathrm{E}>10 \mathrm{GeV})$ behavior of the flux of $\mathrm{CR} \mathrm{e}^{+}$'s where it may be dominated by the decay/annihilation of dark matter particles. We consider in detail, the energy-loss of $\mathrm{e}^{+}$'s above $10 \mathrm{GeV}$ where it is dominated by synchrotron radiation, inverse Compton scattering, triple pair production, and escape from the Galaxy by diffusion in its turbulent magnetic fields. (Other energy loss mechanisms that are important only at energy well below $10 \mathrm{GeV}$, such as Coulomb scattering, ionization and bremsstrahlung, as well as threshold effects, geomagnetic shielding and solar modulation, are not considered in detail, but are included for completeness through a best fit phenomenological depletion factor $\mathrm{D}_{\mathrm{e}^{ \pm}}(\mathrm{E})=1-\exp \left(-(\mathrm{E} / \mathrm{V})^{\alpha}\right)$, which depends on the time of the measurements and on the elecctric charge $( \pm)$ but does not affect the behavior at $\mathrm{E}>10 \mathrm{GeV}$.) Using only standard particle physics, the steady state approximation for CR propagation, and the measured properties of the primary CRs and the local Galactic environment, we show that the spectrum of high energy CR e $^{+}$'s measured by AMS (Aguilar et al. 2014) with high statistics and small systematic errors is consistent with that expected from secondary production of positrons in hadronic interactions of the primary CRs in the local ISM. In particular, the hardening of their spectrum with increasing energy between $\sim 30$ and $150 \mathrm{GeV}$ and its softening beyond $250 \mathrm{GeV}$, as observed with AMS02 (Aguilar et al. 2014), are expected from the transition of their inverse Compton scattering of diffuse Galactic light from the Thomson regime to the Klein-Nishina regime (Schlickeiser \& Ruppel 2010).

Moreover, the break near $1 \mathrm{TeV}$ in the combined $\mathrm{e}^{ \pm}$flux, suggested by the data from H.E.S.S. (Aharonian et al. 2008) may be the maximum re-acceleration energy of electrons in the ISM due to their radiative energy-loss and escape from the Galaxy by diffusion. Indeed a break was reproduced in numerical calculations (e.g., Stawarz et al. 2010), which have used the elaborate GALPROP numerical code (Moskalenko \& Strong 1998a,b). Other standard astroparticle physics interpretations rather than a dark matter annihilation/decay signal are shortly discussed. 


\section{CR Production of positrons in the local ISM:}

The flux of primary Cosmic ray nucleons (free protons and nucleons bound in atomic nuclei) in the energy range between several $\mathrm{GeV}$ and $\mathrm{PeV}$ per nucleon observed near Earth is well described by (e.g., Olive et al. 2014),

$$
\Phi_{\mathrm{p}}(\mathrm{E}) \approx 1.8(\mathrm{E} / \mathrm{GeV})^{-\beta} \mathrm{fu}
$$

where $\beta=2.7$ and $\mathrm{fu}=1 /\left(\mathrm{GeV} \mathrm{cm}^{2} \mathrm{~s} \mathrm{sr}\right)$ is the flux unit. The contribution of nucleons bound in atomic nuclei $(\mathrm{A}, \mathrm{Z})$ to the cosmic ray flux at the same energy per nucleon is less than $5 \%$ and comes mainly from light nuclei whose inelastic cross section per nucleon is $\sim \sigma_{\mathrm{pA}} / \mathrm{A} \approx \sigma_{\mathrm{pp}}$, i.e., roughly that of free protons, while their effective rigidity $(\mathrm{R}=\mathrm{AE} / \mathrm{Z})$ is twice than that of free protons. Hence, due to Feynman scaling (1969), secondary production of high energy positrons through the decay of $\pi$ and $\mathrm{K}$ mesons, which are produced in hadronic collisions of cosmic ray nucleons with the baryons in the local ISM where the mean baryon density is $\mathrm{n}_{\text {ism }} \approx 0.9 \mathrm{~cm}^{-3}$ (Kalberla \& Dedes, 2008) inject into the CR halo a flux of $\mathrm{e}^{+}$'s per unit volume roughly at a rate

$$
\mathrm{J}_{\mathrm{e}^{+}} \approx \mathrm{K}_{\mathrm{e}^{+}} \sigma_{\mathrm{in}}(\mathrm{pp}) \mathrm{c} \mathrm{n}_{\mathrm{ism}} \Phi_{\mathrm{p}}(\mathrm{E})
$$

where $\mathrm{K}_{\mathrm{e}^{+}}$is a constant that depends only on the power-law index of the product $\sigma_{\text {in }}(\mathrm{pp}) \Phi_{\mathrm{p}}(\mathrm{E})$ but not on E itself. In a steady state, the flux $\Phi_{\mathrm{e}^{+}}(\mathrm{E})$ of secondary $\mathrm{e}^{+}$'s produced in the ISM satisfies

$$
\frac{\mathrm{d}}{\mathrm{dE}}\left[\mathrm{b}(\mathrm{E}) \Phi_{\mathrm{e}^{+}}(\mathrm{E})\right]=\mathrm{J}_{\mathrm{e}^{+}}(\mathrm{E})
$$

where $\mathrm{b}(\mathrm{E})=\mathrm{dE} / \mathrm{dt}$ is the loss rate of $\mathrm{e}^{+}$energy by radiation (rad) and by escape (esc) from the Galaxy by diffusion through its turbulent magnetic field. The solution of Eq. (3) for $\Phi_{\mathrm{p}} \propto \mathrm{E}^{-\beta}$ is

$$
\Phi_{\mathrm{e}^{+}}(\mathrm{E}) \approx \mathrm{K}_{\mathrm{e}^{+}} \sigma_{\mathrm{in}}(\mathrm{pp}) \mathrm{n}_{\mathrm{ism}} \mathrm{c} \tau_{\mathrm{e}} \Phi_{\mathrm{p}} /\left(\beta_{\mathrm{j}}-1\right) .
$$

where $\sigma_{\text {in }}(\mathrm{pp}) \approx 30(\mathrm{E} / \mathrm{GeV})^{0.06} \mathrm{mb}, \mathrm{K}_{\mathrm{e}^{+}} \approx 7 \times 10^{-3}$ for $\beta_{j} \approx \beta-0.06=2.64$, and $\tau_{\mathrm{e}}=\mathrm{E} /(\mathrm{dE} / \mathrm{dt})$ is the mean life-time of positrons in the ISM due to their escape (esc) from the Galaxy by diffusion and radiative (rad) energy losses (inverse Compton scattering of background photons and synchrotron radiation). Hence, the expected flux of high energy CR $\mathrm{e}^{+}$'s near Earth is simply

$$
\Phi_{\mathrm{e}^{+}}(\mathrm{E}) \approx 6.22 \times 10^{-18} \mathrm{E}^{-2.64} \tau_{\mathrm{e}}(\mathrm{E}) f u .
$$

where the combined radiative losses and escape by diffusion yields

$$
1 / \tau_{\mathrm{e}}=1 / \tau_{\mathrm{esc}}+1 / \tau_{\mathrm{rad}}
$$


At high energy, the rigidities of $\mathrm{e}^{+}$'s and protons become practically equal. Consequently, their escape times by diffusion become practically equal. For random Galactic magnetic fields with a Kolmogorov spectrum

$$
\tau_{\mathrm{esc}} \approx(7.5 \pm 1.5) \times 10^{14}(\mathrm{E} / \mathrm{GeV})^{-1 / 3} \mathrm{~s}
$$

where the normalization has been adjusted to the value obtained by Lipari (2014) from a leaky box model analysis of the flux ratio ${ }^{10} \mathrm{Be} /{ }^{9} \mathrm{Be}$ measured with the Cosmic Ray Isotope Spectrometer (CRIS) in the energy range 70-145 MeV/nucleon (Yanasak et al. 2001). At the $\mathrm{CR}$ ankle near $\mathrm{E} \approx 4 \times 10^{9} \mathrm{GeV}$, this normalization yields $\tau_{\mathrm{esc}}=(15 \pm 3) \mathrm{ky}$, consistent with an expected free escape time $\mathrm{H} / \mathrm{c}=5 \pm 1 \mathrm{kpc} / \mathrm{c}=(16.3 \pm 3.2) \mathrm{ky}$, where $\mathrm{H}$ is the typical scale height of the Galactic cosmic ray halo perpendicular to the Galactic disk (e.g., Trotta et al. 2011).

Synchrotron emission and inverse Compton scattering of background photons in the Thomson regime yield

$$
\tau_{\text {rad }} \approx \frac{3\left(\mathrm{~m}_{\mathrm{e}} \mathrm{c}^{2}\right)^{2}}{4 \sigma_{\mathrm{T}} \mathrm{c} \mathrm{UE}} \approx 0.67 \times 10^{16}(\mathrm{E} / \mathrm{GeV})^{-1} \mathrm{~s}
$$

where $\sigma_{\mathrm{T}} \approx 0.66$ barn is the Thomson cross section and $\mathrm{U} \sim 1.47 \mathrm{eV} / \mathrm{cm}^{3}$ is the energy density of the background radiations plus the magnetic field in the local ISM. Roughly, the magnetic field $\mathrm{B} \sim 4 \mu \mathrm{G}$ in the local ISM contributes $\mathrm{B}^{2} / 8 \pi \sim 0.40 \mathrm{eV} / \mathrm{cm}^{3}$ to $\mathrm{U}$, the diffuse Galactic light (DGL) contributes $\sim 0.39 \mathrm{eV} / \mathrm{cm}^{3}$, the far infrared (FIR) light contributes $\sim 0.40 \mathrm{eV} / \mathrm{cm}^{3}$, and the cosmic microwave background (CMB) contributes $0.26 \mathrm{eV} / \mathrm{cm}^{3}$ (e.g., Porter et al. 2006; Schlickeiser \& Ruppel 2010).

In the Klein-Nishina regime, $\left(\mathrm{w}=4 \mathrm{E}_{\mathrm{e}} \epsilon_{\gamma} /\left(\mathrm{m}_{\mathrm{e}} \mathrm{c}^{2}\right)^{2} \sim 1\right)$, the energy loss-rate by ICS is given by (e.g., Blumenthal \& Gould 1970)

$$
\frac{\mathrm{dE}}{\mathrm{dt}} \approx-\frac{3 \sigma_{\mathrm{T}} \mathrm{c} \mathrm{U} \mathrm{U}_{\gamma}\left(\mathrm{m}_{\mathrm{e}} \mathrm{c}^{2}\right)^{2}}{8 \epsilon_{\gamma}^{2}}[\operatorname{lnw}-11 / 6] .
$$

Consequently, as can be seen from Eq. (3), in a steady state, an injected power-law spectrum of electrons becomes softer in the Thomson regime, $\mathrm{E}^{-\beta_{\mathrm{j}}} \rightarrow \mathrm{E}^{-\beta_{\mathrm{j}}-1}$, whereas in the KleinNishina regime it becomes harder, $\mathrm{E}^{-\beta_{\mathrm{j}}} \rightarrow \mathrm{E}^{-\beta_{\mathrm{j}}+1}$.

In the case of a gray body radiation of temperature $\mathrm{T}$, the transition from the Thomson regime to the Klein-Nishina regime can be interpolated through replacing $\mathrm{U}(\mathrm{E})$ by

$$
\mathrm{U}_{\mathrm{KN}}(\mathrm{E}) \approx \mathrm{U}(\mathrm{E}) \mathrm{E}_{\mathrm{KN}}^{2} /\left(\mathrm{E}^{2}+\mathrm{E}_{\mathrm{KN}}^{2}\right)
$$

where (Schlickeiser \& Ruppel 2010)

$$
\mathrm{E}_{\mathrm{KN}} \approx \frac{0.27\left(\mathrm{~m}_{\mathrm{e}} \mathrm{c}^{2}\right)^{2}}{\mathrm{kT}}
$$


with $\mathrm{k}$ being the Boltzman constant. For the local DGL, $\mathrm{T} \approx 5700^{\circ} \mathrm{K}$ and $\mathrm{E}_{\mathrm{KN}} \approx 140 \mathrm{GeV}$. For the Galactic FIR radiation and the CMB, $\mathrm{E}_{\mathrm{KN}}$ is well above $10 \mathrm{TeV}$.

Since the estimated values of the local energy densities of the DGL and FIR, as well as the local B suffer from uncertainties, we have best fitted their values within the errors of their estimated values. At $100 \mathrm{GeV}$, they yield for instance, $\tau_{\text {esc }}=1.62 \times 10^{14} \mathrm{~s}$, $\tau_{\text {rad }} \approx 7.2 \times 10^{13} \mathrm{~s}$, and consequently $\tau_{\mathrm{e}} \approx 0.50 \times 10^{14} \mathrm{~s} \approx 1.6 \mathrm{MY}$.

The flux of high energy $\mathrm{e}^{+}$CRs measured with AMS02 (Aguilar et al. 2014) and the flux expected from CR production of $\mathrm{e}^{+}$'s in the ISM as given by Eq. (5) multiplied by the low-energy best fit phenomenological depletion factor $\mathrm{D}_{\mathrm{e}^{+}}(\mathrm{E})=1-\exp \left(-(\mathrm{E} / \mathrm{V})^{\alpha}\right)$ with $\mathrm{V}=3.90 \mathrm{GeV}$ and $\alpha=1.78$ are compared in Fig. 1 .

\section{Origin of the $\mathbf{C R} \mathrm{e}^{-}$flux}

Most of the Galactic accelerators of high energy cosmic rays (supernova remnants, pulsars, gamma ray bursts, and the massive black hole near the Galactic center) are located in the Galactic disk within $\sim 4 \mathrm{kpc}$ from its center (e.g., Case \& Bhattacharya 1998; Yusifov, \& I. Kucuk 2004; Lorimer et al. 2006). Presumably, the Fermi acceleration of high energy electrons, protons and nuclei is simultaneous and imparts to them the same Lorentz factor distribution (e.g. Dar \& De Rújula 2008), i.e., a source distribution $\mathrm{J}_{\mathrm{e}^{-}} \propto \mathrm{J}_{\mathrm{p}}$. These primary CRs can reach Earth from their common sources by diffusion (dif) through the turbulent Galactic magnetic fields. High energy CR protons and electrons have practically the same rigidity (except for the sign). Consequently, without energy losses, they would have reached Earth from their common sources after the same mean diffusion time, and their fluxes in the local ISM then would have satisfied $\phi_{\mathrm{e}^{-}}(\mathrm{E}) \propto \Phi_{\mathrm{p}}(\mathrm{E})$. This relation can hold as long as their energy loss by radiation is quite small during their diffusion time from source to Earth.

The radiative energy loss of $\mathrm{e}^{-} \mathrm{CRs}$ in the ISM satisfies $\mathrm{dE} / \mathrm{dt}=-\mathrm{b} \mathrm{E}^{2}$ whose solution is $E=E_{0}\left[1-b E t_{d i f}\right]$ where $E$ is the observed energy of the $\mathrm{e}^{-} \mathrm{CR}$ near Earth and $\mathrm{E}_{0}$ is its injection energy. It implies that most of the sources that are located beyond $\sim 4 \mathrm{kpc}$ cannot

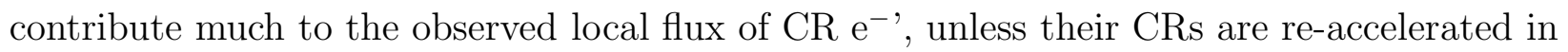
the ISM or transported ballistically over long Galactic distances by highly relativistic jets, such as those emitted in supernova explosions of Type Ic, most of which do not point in our direction (e.g., Dar \& Plaga 1999, Dar \& De Rújula 2008).

If the escape time of CR protons and electrons from their sources into the ISM is much shorter than their energy loss rate there, then the Fermi accelerated high energy electrons are injected into the ISM at a rate $\mathrm{J}_{\mathrm{e}} \propto \Phi_{\mathrm{p}} / \tau_{\mathrm{esc}}(\mathrm{E})$. Hence, their steady state flux near Earth 
satisfies

$$
\Phi_{\mathrm{e}^{-}}=\mathrm{D}_{\mathrm{e}^{-}}(\mathrm{E}) \mathrm{A}_{\mathrm{e}^{-}} \mathrm{E}^{-\beta} \tau_{\mathrm{e}} / \tau_{\mathrm{esc}}+0.74 \Phi_{\mathrm{e}^{+}}
$$

The first term on the right hand side (RHS) of Eq. (12) is due to primary CR $\mathrm{e}^{-}$'s, presumably re-accelerated in the ISM or transported ballistically by highly relativistic jets from all distant sources together with the $\mathrm{CR}$ protons $\left(\mathrm{A}_{\mathrm{e}^{-}}\right.$is an unknown flux normalization constant, which is treated as a free parameter.) The second term on the RHS is the contribution from hadronic production of high energy $\mathrm{e}^{-}$'s in pp collisions, in the local ISM assuming an inclusive production ratio identical to that of muons, $\mathrm{e}^{-} / \mathrm{e}^{+} \approx \mu^{-} / \mu^{+}=0.74$, which was measured for atmospheric muons and in accelerator experiments (Haino et al. 2004; Archard et al. 2004; Adamson et al. 2007; Khachatryan et al. 2010; Agafonova et al. 2010). Eq. (12) can also be written as

$$
\mathrm{E}^{3} \Phi_{\mathrm{e}^{-}}=\frac{\mathrm{D}_{\mathrm{e}^{-}}(\mathrm{E}) \mathrm{A}_{\mathrm{e}^{-}} \mathrm{E}^{3-\beta}}{\left(1+\tau_{\mathrm{esc}} / \tau_{\mathrm{rad}}\right)}+0.74 \mathrm{E}^{3} \Phi_{\mathrm{e}^{+}},
$$

Assuming that the mean radiation field in the CR halo can be represented by that in the solar neighborhood, the predicted flux of $\mathrm{CR} \mathrm{e}^{-}$'s as given by Eq. (13) for the best fit values $\mathrm{A}_{\mathrm{e}^{-}}=0.27 \mathrm{GeV}^{3} \mathrm{fu}, \mathrm{V}=2.25 \mathrm{GeV}$ and $\alpha=1.46$ is compared in Fig. 2 to the $\mathrm{CR}$ $\mathrm{e}^{-}$flux measured with AMS02 (Aguilar et al. 2014). The agreement is quite satisfactory $\left(\chi^{2} / \mathrm{df}=0.85\right)$.

\section{The $\mathrm{e}^{+}$fraction and the combined $\mathrm{e}^{ \pm}$flux}

The positron fraction $\Phi_{\mathrm{e}^{+}} / \Phi_{\mathrm{e}}$ as a function of energy obtained from our predicted $\mathrm{e}^{ \pm}$ fluxes, which are plotted in Figs. 1 and 2, and the positron fraction measured with AMS02 (Accardo et al. 2014) near Earth are compared in Fig. $3\left(\chi^{2} / \mathrm{df}=0.35\right)$. The combined $\mathrm{e}^{ \pm}$flux

$\Phi_{\mathrm{e}}=\Phi_{\mathrm{e}^{+}}+\Phi_{\mathrm{e}^{-}}$measured near Earth with AMS02 (Aguilar et al. 2014) and the expected flux obtained from standard astroparticle physics are compared in Fig. $4\left(\chi^{2} / \mathrm{df}=1.27\right)$. As can be seen from Figs. 1-4, the agreement between the near Earth $\mathrm{e}^{ \pm} \mathrm{CR}$ fluxes measured with AMS02 and those expected from standard astroparticle physics is quite good.

\section{Dark matter annihilation signal in AMS02 ?}

The AMS collaboration presented an impressive 'minimal model' fit $\left(\chi^{2} / \mathrm{df}=0.63\right)$ to their updated data (Accardo et al. 2014) on the CR positron fraction, which is shown in Fig. 5. In this model the $\mathrm{e}^{ \pm} \mathrm{CR}$ fluxes were parametrized as the sum of a power law spectrum and a common source term with an exponential cutoff, which may represent a 
dark matter decay/annihilation contribution,

$$
\begin{aligned}
& \Phi_{\mathrm{e}^{+}}=\mathrm{C}_{\mathrm{e}^{+}} \mathrm{E}^{-\gamma_{\mathrm{e}^{+}}}+\mathrm{C}_{\mathrm{s}} \mathrm{e}^{-\mathrm{E} / \mathrm{E}_{\mathrm{s}}}, \\
& \Phi_{\mathrm{e}^{-}}=\mathrm{C}_{\mathrm{e}^{-}} \mathrm{E}^{-\gamma_{\mathrm{e}^{-}}}+\mathrm{C}_{\mathrm{s}} \mathrm{e}^{-\mathrm{E} / \mathrm{E}_{\mathrm{s}}},
\end{aligned}
$$

with $\mathrm{E}$ in $\mathrm{GeV}$. However, the positron fraction $\Phi_{\mathrm{e}^{+}} /\left(\Phi_{\mathrm{e}^{-}}+\Phi_{\mathrm{e}^{+}}\right)$is invariant under division of both fluxes by an arbitrary E-dependent function. In particular, by dividing the minimal model fluxes by $\mathrm{C}_{\mathrm{e}^{+}} \mathrm{E}^{-\gamma_{\mathrm{e}^{+}}}$, a very good best fit $\left(\chi^{2} / d f=36.4 / 58\right)$ to the positron fraction in the energy range 1 to $500 \mathrm{GeV}$ yielded (Accardo et al. 2014) $\mathrm{C}_{\mathrm{e}^{+}} / \mathrm{C}_{\mathrm{e}^{-}}=0.091 \pm 0.001$, $\gamma_{\mathrm{e}^{+}}-\gamma_{\mathrm{e}^{-}}=0.56 \pm 0.03, \mathrm{C}_{\mathrm{s}} / \mathrm{C}_{\mathrm{e}^{-}}=0.0061 \pm 0.0009, \gamma_{\mathrm{s}}-\gamma_{\mathrm{e}^{-}}=-0.72 \pm 0.04$ and a cutoff parameter $\mathrm{E}_{\mathrm{s}}=0.54 \pm 0.17 \mathrm{TeV}$. These best fit parameters specify the $\mathrm{e}^{ \pm}$fluxes of the minimal model only up to an unknown common power-law factor $\mathrm{C}_{\mathrm{e}^{+}} \mathrm{E}^{-\gamma_{\mathrm{e}}+}$.

A major feature of the minimal model is the sharp decline of the positron fraction above $\sim 500 \mathrm{GeV}$. In order to test the AMS minimal model, we have best fitted $\Phi_{\mathrm{e}^{+}}$and $\Phi_{\mathrm{e}^{-}}$as given by Eqs. (13) and (14), respectively, to the separate $\mathrm{e}^{+}$and $\mathrm{e}^{-}$fluxes measured with AMS02 (Aguilar et al. 2014). The best fit parameters were constrained to reproduce the above relations between the parameters of the minimal model. Unlike the minimal model impressive fit to the positron fraction published by the AMS collaboration, (Accardo et al. 2014) as shown in Fig. 5, the best fits to the separate $\mathrm{e}^{+}$and $\mathrm{e}^{-}$fluxes, and to their sum, are rather poor, as shown in Figs. 6-8. That is true in particular for $\mathrm{E}<10 \mathrm{GeV}$ and $\mathrm{E}>300$ $\mathrm{GeV}$.

One may argue, however, that the minimal model represents well the source spectra of $e^{ \pm}$CRs, which are later modified by propagation effects in/near source, in the ISM and in the heliosphere. But, because solar modulation, escape by diffusion in the turbulent Galactic magnetic fields, and energy-loss through ionization, bremsstrhalung, synchrotron radiation, inverse Compton scattering and pair production, which strongly affect the observed spectra of $e^{ \pm}$CRs near Earth, are independent of the sign of charge, they modify the injection spectra of $e^{ \pm}$by the same energy-loss factor $\mathrm{E} /(\mathrm{dE} / \mathrm{dt})=\mathrm{b}(\mathrm{E})$. However, the spatial distribution of Galactic dark matter is quite different from that of the more conventional sources of primary $\mathrm{e}^{-}$and secondary $\mathrm{e}^{ \pm}$CRs. This makes rather unlikely the possibility that the strong modifications of the source spectra of both high energy $\mathrm{e}^{+}$and $\mathrm{e}^{-}$CRs observed near Earth are nearly identical.

\section{6. $\quad \mathrm{CR}^{ \pm}$knee near TeV?}

The energy spectrum of high energy $\mathrm{e}^{ \pm}$CRs measured with H.E.S.S. (Aharonian et al. 2008,2009) suggests a sharp break in the combined $\mathrm{e}^{ \pm}$spectrum near E $\sim \mathrm{TeV}$. This is 
shown in Fig. 10 where we plotted a smooth cutoff power-law fit to the combined $\mathrm{e}^{ \pm}$flux measured with AMS02 (Aguilar et al. 2014) and with H.E.S.S (Aharonian et al 2008, 2009) after normalizing the H.E.S.S data within their reported systematic errors to match the more precise AMS02 sub-TeV data. This fit yield a break energy around $1 \mathrm{TeV}$.

Fermi acceleration of electrons is cut-off by synchrotron emission when the energy loss rate by synchrotron radiation exceeds the energy gain by magnetic deflections. This synchrotron cutoff energy is given roughly by $\mathrm{E} \approx \sqrt{6 \mathrm{e} / \sigma_{\mathrm{T}} \mathrm{B}} \mathrm{m}_{\mathrm{e}} \mathrm{c}^{2}$. However, the typical magnetic field B in supernova remnants (SNRs), which are widely accepted as the main source of high energy Galactic CR electrons, is well below $100 \mu \mathrm{G}$. The corresponding synchrotron cutoff in the energy spectrum of CR e's accelerated in SNRs is expected at $\gtrsim 1 \mathrm{PeV}$, well above that suggested by the H.E.S.S. data.

The observed flux of primary $\mathrm{e}^{-}$CRs is also cut-off when their radiative life becomes shorter than their travel time by diffusion from source to Earth as discussed in Section 3. However, for the main Galactic sources, this cut-off is much below the H.E.S.S. break/cut-off.

The H.E.S.S. cutoff could be a dark matter signal. However, the data of Fermi-LAT (Ackermann et al. 2010) and AMS02 (Aguilar et al. 2014) published so far, do not show any hint for such a break below TeV. Moreover, such a break, if real, still could be explained by standard astroparticle physics rather than as a dark matter signal. The origin of a break could be, e.g.,

(A) A re-acceleration cutoff when the re-acceleration time in the Galactic ISM exceeds the electrons' life time due to their radiative energy losses and escape from the Galaxy by diffusion.

(B) $\mathrm{CR}^{-}$"knee" at $\mathrm{E}_{\text {knee }}\left(\mathrm{e}^{-}\right)=\left(\mathrm{m}_{\mathrm{e}} / \mathrm{m}_{\mathrm{p}}\right) \mathrm{E}_{\text {knee }}(\mathrm{p}) \approx 1 \mathrm{TeV}$ in the spectrum of $\mathrm{e}^{-} \mathrm{CRs}$, which are Fermi accelerated together with protons and nuclei by the highly relativistic jets launched in SNe Ic to the same Lorentz factor distribution (e.g., Dar and De Rújula 2008). ( $\mathrm{E}_{\mathrm{knee}}(\mathrm{p}) \approx 2$ $\mathrm{PeV}$ is the CR "knee" in the flux per nucleon of CR nuclei).

(C) A cutoff when the energy deposition rate of high energy electrons in the ISM by highly relativistic jets cannot compensate anymore their energy loss by radiation and escape from the Galaxy by diffusion.

For other possible standard astroparticle physics origins of the H.E.S.S break see, e.g., Stawarz et al. (2010) and Cowsik et al. (2014). 


\section{Conclusions}

Figs. 1-4 demonstrate that standard astroparticle physics, such as positron production in the ISM in hadronic collisions of the primary CRs with gas in the local ISM and electron acceleration in a variety of Galactic sources, can explain the observed fluxes of CR electrons and positrons near Earth, which were measured with AMS02 (Accardo et al. 2014; Aguilar et al. 2014) without invoking a contribution from annihilation/decay of dark matter particles. In particular, the hardening of the positron flux between 20 and $200 \mathrm{GeV}$ can be due to the Klein-Nishina supression of the energy loss of positrons by inverse Compton scattering from the diffuse Galactic light in the local ISM.

Neither the current published data on the spectra of the separate and combined $\mathrm{e}^{ \pm} \mathrm{CRs}$ near Earth, nor the minimal model fit by the AMS collaboration to their measured positron fraction (Accardo et al. 2014) provide compelling evidence or hints for a contribution from decay or annihilation of dark matter particles.

If hadronic production of mesons in the local ISM at energies well above TeV becomes the main source of high energy $\mathrm{e}^{ \pm}$CRs near Earth, then the positron fraction should reach there the asymptotic value $\approx 0.57$ (Dado \& Dar 2010; Cowsik et al. 2010) expected from the observed charge ratio $\mu^{+} / \mu^{-} \approx 1.35$ of high energy atmospheric and accelerator muons.

The existence of a break near $\mathrm{TeV}$ in the combined $\mathrm{e}^{ \pm}$flux measured with H.E.S.S., still needs confirmation from independent and more precise measurements with instruments such as Fermi-LAT and the AMS. If verified, standard astroparticle physics may still explain it as shortly discussed in Section 6. Moreover, any alternative interpretation of its origin, including dark matter, must provide falsifiable predictions to establish its validity.

\section{REFERENCES}

Accardo, L., Aguilar, M., Aisa, D., et al. (AMS Collab.) 2014, PRL, 113, 121101

Ackermann, M., Ajello, M., Atwood, W. B., et al. (Fermi-LAT Collab.) PRD, 2010, 82, 092004 (arXiv:1008.3999)

Ackermann, M., Ajello, M., Allafort, A., et al. (Fermi-LAT Collab.), 2012, PRL, 011103 (arXiv:1109.0521)

Adamson, P., Andreopoulos, C., Arms, K. E., et al. 2007, Phys. Rev. D76, 052003 (arXiv:0705.3815) 
Ade, P. A. R., Aghanim, N., Armitage-Caplan, C., et al (Planck Collab.) 2014, A\&A, 571, 16 (arXiv:1303.5076)

Adriani, O., Barbarino, G. C., Bazilevskaya, G. A., et al. (PAMELA Collab.) 2009, Nature, 458, 607 (arXiv:0810.4995)

Adriani, O., Barbarino, G. C., Bazilevskaya, G. A., et al. (PAMELA Collab.) 2010, Astropart. Phys. 34, 1

Adriani, O., Barbarino, G. C., Bazilevskaya, G. A., et al. (PAMELA Collab.), 2013, PRL, 111, 081102 (arXiv:1308.0133)

Agafonova, N., A. Anokhina, A., Aoki, S., et al.(OPERA Collab.) 2010, Eur. Phys. J. C67, 25 (arXiv:1003.1907)

Aguilar, M., Alberti, G., Alpat, B., et al. (AMS Collab.), 2013, PRL, 110, 141102

Aguilar, M., Aisa, D., Alvino, A., et al. (AMS Collab.) 2014a, PRL, 113, 121102

Aguilar, M., Aisa, D., Alpat, B., et al. (AMS Collab.) 2014b Phys. Rev. Lett. 113, 221102

Aharonian, F., Akhperjanian, A. G., Barres de Almeida, U., et al. (H.E.S.S. Collab.) 2008, PRL, 101, 261104

Aharonian, F., Akhperjanian, A. G., Anton, G., et al. (H.E.S.S. Collab.) 2009, A\&A, 508, 561

Archard, P., Adriani, O., Aguilar-Benitez, M., et al. (L3 collab.) 2004, Phys. Lett. B, 598, 15

Arkani-Hamed, N., Finkbeiner, D. P., Slatyer, T. R., Weiner, N., 2009, Phys. Rev. D79, 015014 arXiv:0810.0713

Bennett, C. L., Larson, D., Weiland, J. L., et al. 2013, ApJS, 208, 20 (arXiv:1212.5225)

Bergstrom, L., Bringmann, T., Edsjo, J., 2008, PRD, 78, 103520 (arXiv:0808.3725)

Bergstrom, L., 2009, New J. Phys. 11, 105006 (arXiv:0903.4849)

Bergstrom, L., Edsjo, J., Zaharijas, G. 2009, Phys. Rev. Lett. 103, 031103 (arXiv:0905.0333)

Bergstrom, L., et al. 2013, Phys. Rev. Lett. 111, 1101 (arXiv:1306.3983)

Blasi, P., 2009, Phys. Rev. Lett. 103, 051104 (arXiv:0903.2794) 
Blum, K., Katz, B., Waxman, E., 2013, Phys. Rev. Lett. 111, 211101 ([arXiv:1305.1324]

Blumenthal, G. R., Gould, R. J., 1970, RMP, 42, 237

Case, G. L., Bhattacharya, D. 1998, Astrophys. J, 504, 761 (arXiv:astro-ph/9807162)

Chang, J., Adams, J. H., Ahn, H. S., et al. (ATIC collab.), Nature, 2008, 456, 362

Cholis, I., Goodenough, L., Hooper, D., Simet, M., Weiner, N., 2009 Phys. Rev. D80, 123511 (arXiv:0809.1683)

Cirelli, M., Kadastik, M., Raidal, M., Strumia, A., 2009, Nucl. Phys. B813 (arXiv:0809.2409)

Cowsik, R.\& Burch, B., 2010, Phys. ReV. D82, 023009 (arXiv:0908.3494)

Cowsik, R., Burch, B., Madziwa-Nussinov, T., 2014, ApJ, 786, 124 (arXiv:1305.1242)

Dado, S., Dar, A., 2010, Mem. Soc. Ast. It. 81, 132 (arXiv:0903.0165)

Dar, A., De Rújula, A., 2008, Phys. Rep. 466, 179 (arXiv:hep-ph/0606199)

Dar, A., Plaga, R., 1999 A\&A 349, 259 (arXiv:astro-ph/9902138)

Fermi, E., 1949, PR, 75, 1169

Feynman, R. P., 1969, PRL ,23, 1415

Haino, S., Sanuki, T., Abe, K., et al. (BESS Collab.) 2004, Phys. Lett. B594, 35 (arXiv:astro-ph/0403704)

Hooper, D, Blasi, P., Serpico, P. D., 2009, JCAP, 01, 25 (arXiv:0810.1527)

Ibarra, A., Tran, D., Weniger, C., 2013, Int. J. Mod. Phys. A, 28, 1330040 for a recent review, and references therein (arXiv:1307.6434)

Jungman, G., Kamionkowski, M., Griest, K., 1996, Phys. Rep. 267, 195 (arXiv:hep-ph/9506380)

Kalberla, P. M. W., Dedes, L., 2008, A\&A, 487, 951 (arXiv:0804.4831)

Kolmogorov, A., 1941, Dokl. Akad. Nauk SSSR, 30, 301 reprinted in Proc. R. Soc. London A $434,(1941)$

Khachatryan, V., Sirunyan, A. M., Tumasyan, A., et al.(CMS Collab.) 2010, Phys. Lett. B692, 83 (arXiv:1005.5332) 
Linden, T., Profumo, S., 2013, ApJ, 772, 18 (arXiv:1304.1791)

Lipari, P., 2014, arXiv1407.5223

Moskalenko, I. V., Strong, A. W., 1998, ApJ, 493, 694 (arXiv:astro-ph/9807150)

Moskalenko, I.V. \& Strong, A. W., 1998, Astrophys. J. 509, 212 (arXiv:astro-ph/9807150). See also http://galprop.stanford.edu for the latest upgrade.

Olive, K., Agashe, K., Amsler, C., et al. (PDG Collab.) 2014, Chin. Phys. C38, 090001

Pohl, M., 2009, Phys. Rev. D79, 041301 (arXiv:0812.1174)

Pohl, M., 2014, arXiv:1412.2482

Porter, T. A. Moskalenko, I. V., Strong, A. W. 2006, ApJ, 648, L29 (astro-ph/0607344)

Rubin, V. C., Ford, Jr. W. K., 1970, ApJ, 159, 379403

Shaviv, N. J., Nakar, O. E., Piran, T., 2009, PRL, 103, 111302 (arXiv:0902.0376)

Schlickeiser, R., Ruppel, J., 2010, NJP, 12, 033044

Stawarz, L., Petrosian, V., Blandford, R. D., 2010, ApJ. 710, 236 (arXiv:0908.1094).

Trotta, R., G. Johannesson, G., Moskalenko, I. V., et al. 2011, ApJ, 729, 106 (arXiv:1011.0037)

Yanasak, N. E., Wiedenbeck, M. E., Mewaldt, R. A., et al. (CRIS Collab.) 2001, ApJ, 563, 768

Yusifov, I., Kucuk, I. 2004, A\&A 422, 545 (arXiv:astro-ph/0405559)

Zwicky, F., 1933, Helvetica Physica Acta, 6, 110

Zwicky, F., 1937, Helvetica Physica Acta, 1937, ApJ, 86, 217 


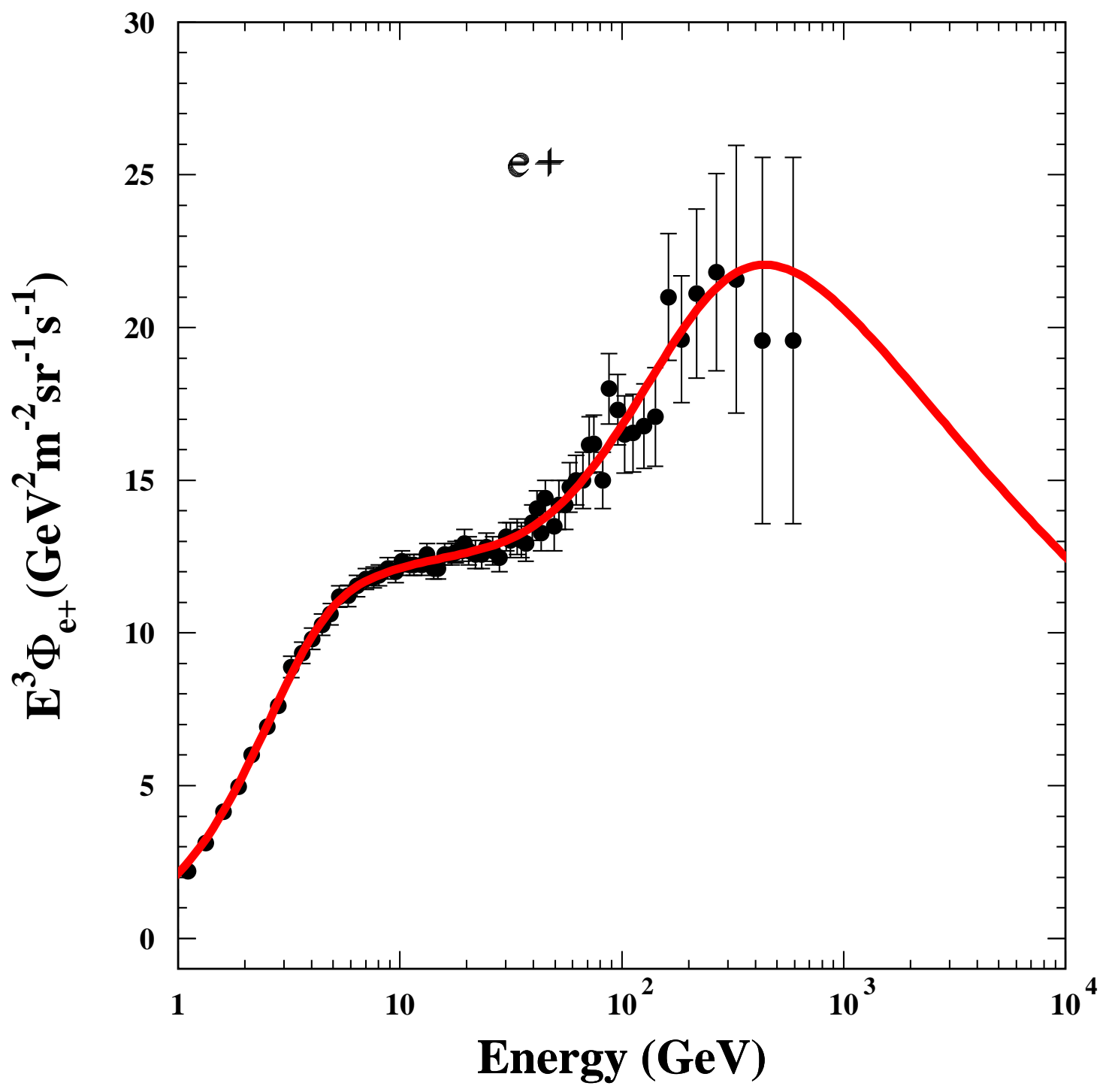

Fig. 1.- Comparison between the high energy $\mathrm{e}^{+}$CR flux measured with AMS02 (Aguilar et al. 2014) and the secondary $\mathrm{e}^{+}$flux expected from hadronic interactions of the primary CR nucleons in the local ISM. 


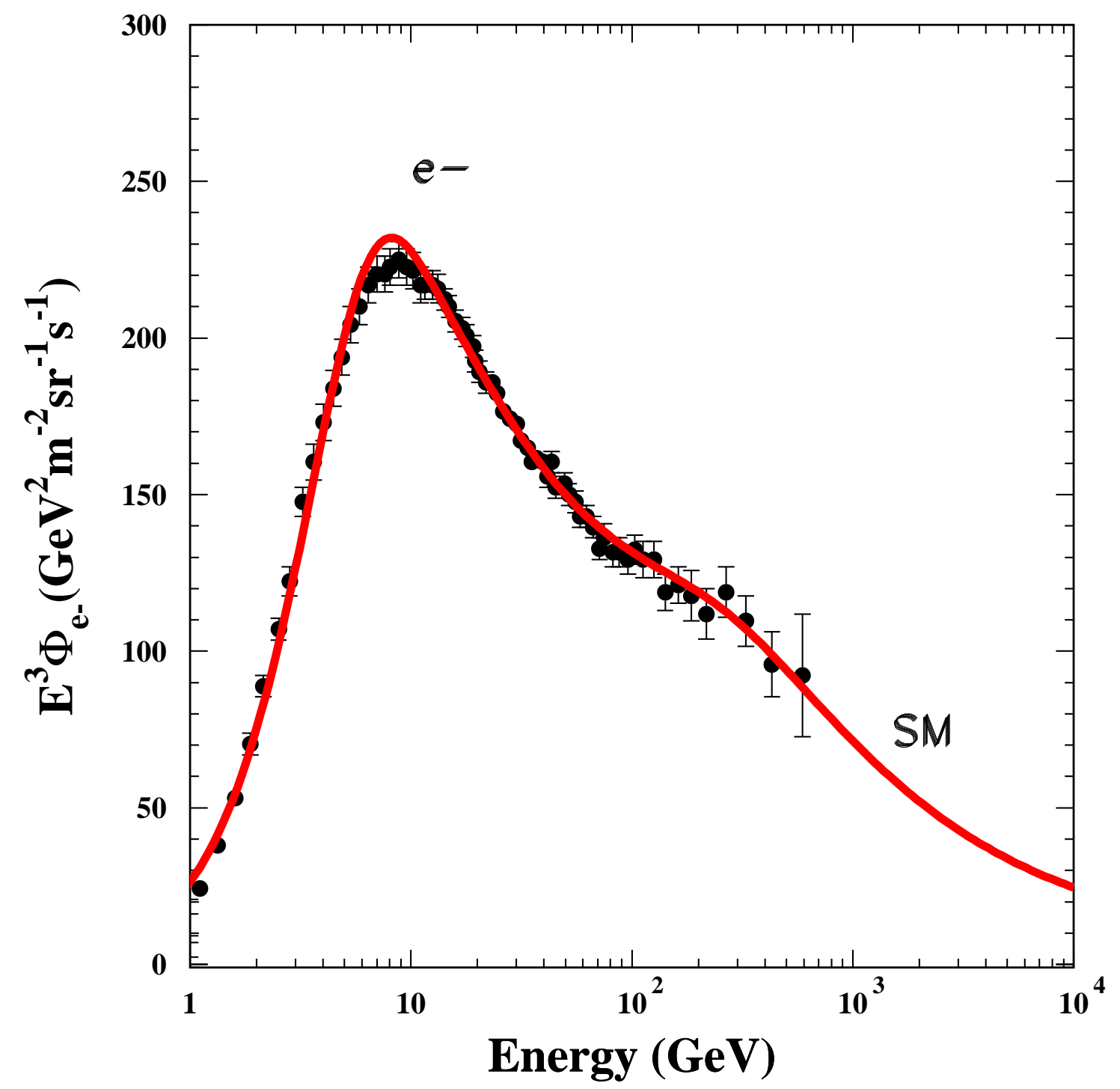

Fig. 2.- Comparison between the flux of high energy e ${ }^{-}$CRs measured with AMS02 (Aguilar et al. 2014) and the flux expected from Fermi acceleration in source of $\mathrm{e}^{-}$'s plus secondary production in/near source and in the ISM. 


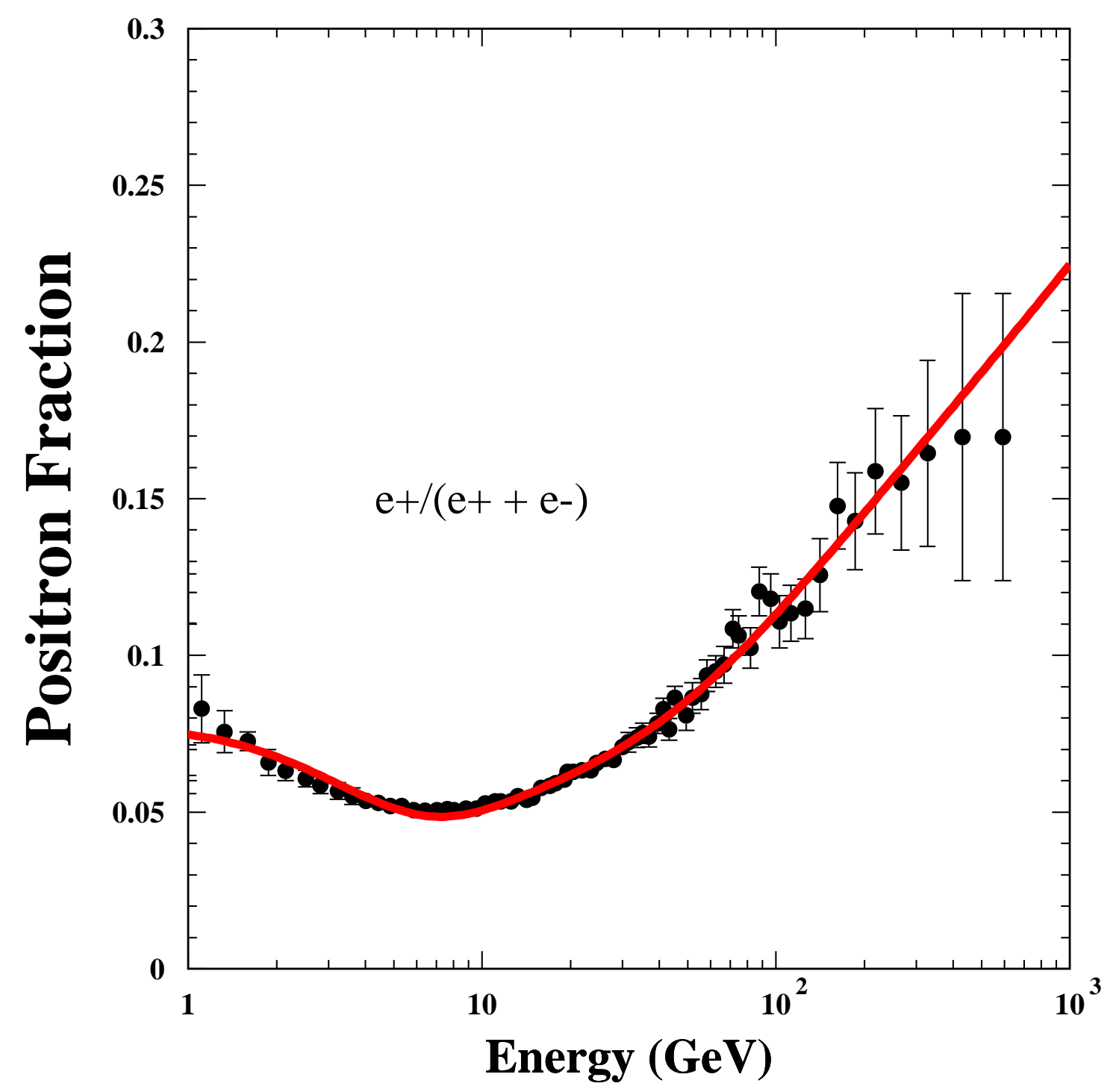

Fig. 3.- Comparison between the CR positron fraction near Earth as measured with AMS02 (Accardo et al. 2014) and the positron fraction obtained from the predicted $\mathrm{e}^{ \pm} \mathrm{CR}$ fluxes shown in Figs. 1 and 2. 


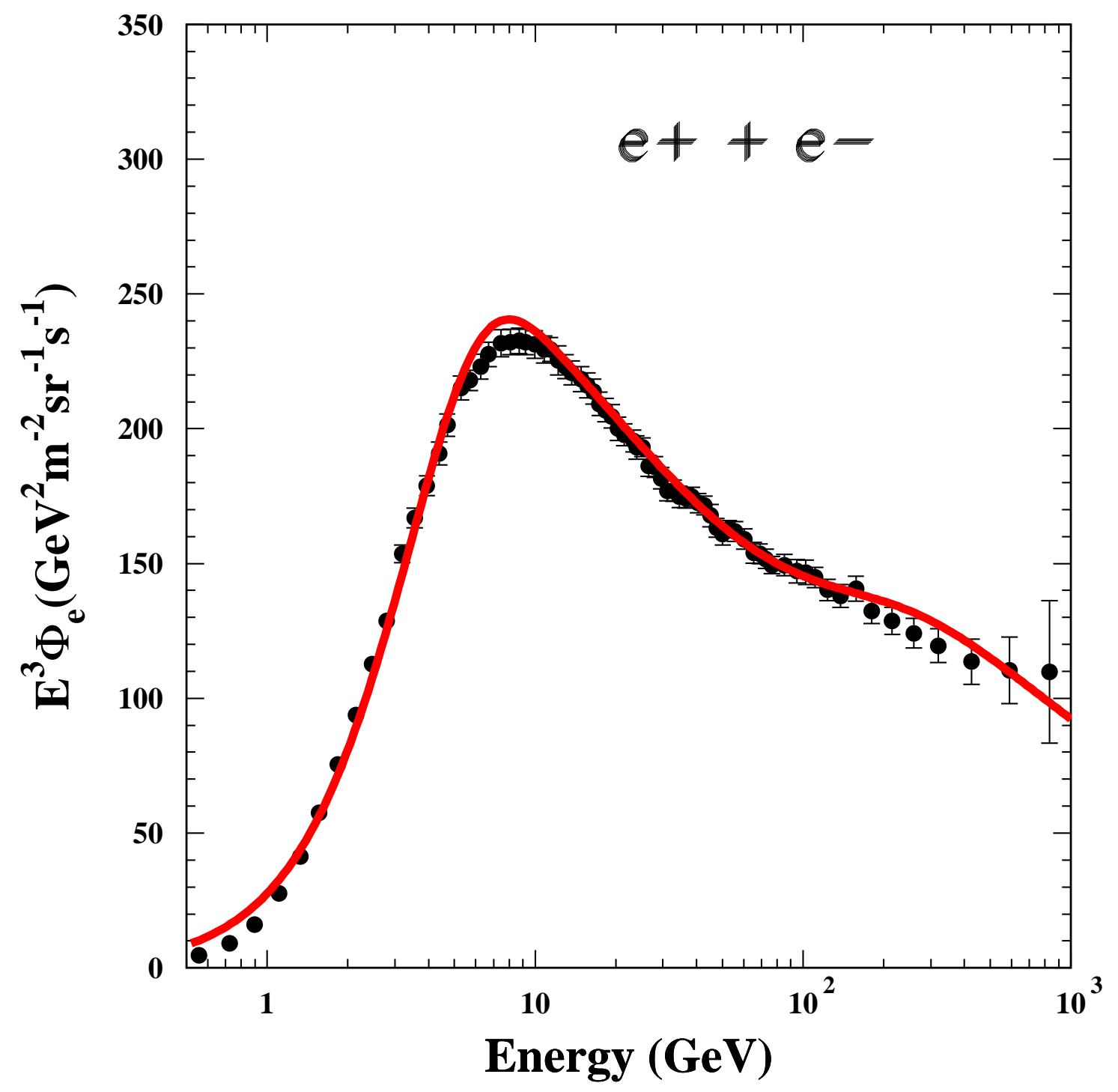

Fig. 4.- Comparison between the combined $\mathrm{e}^{ \pm}$CR flux measured with AMS02 (Aguilar al. 2014) and the flux obtained from our standard particle astrophysics model. 


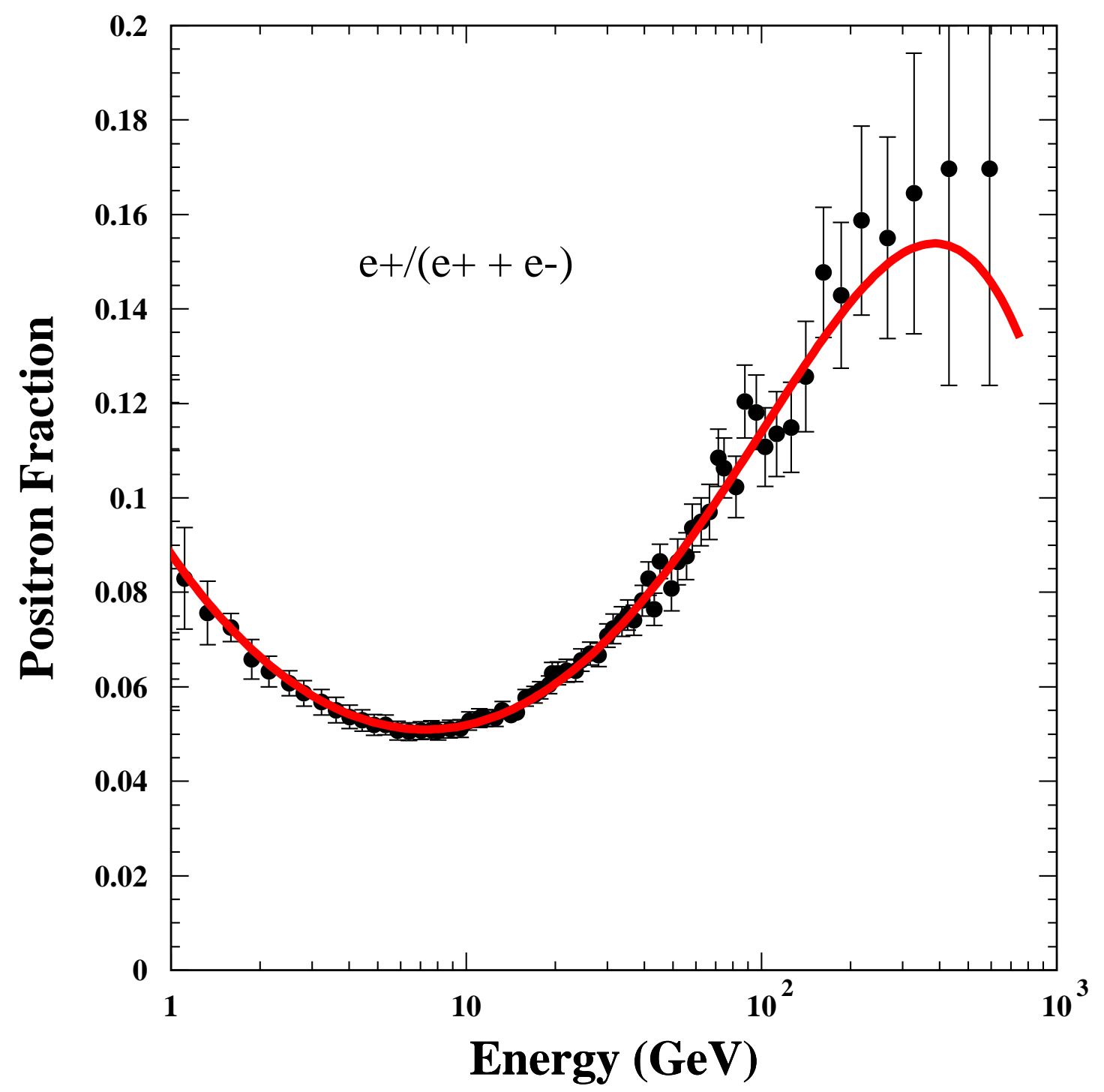

Fig. 5.- The best fit 'minimal model' positron fraction (Accardo et al. 2014) to the positron fraction measured with AMS02. 


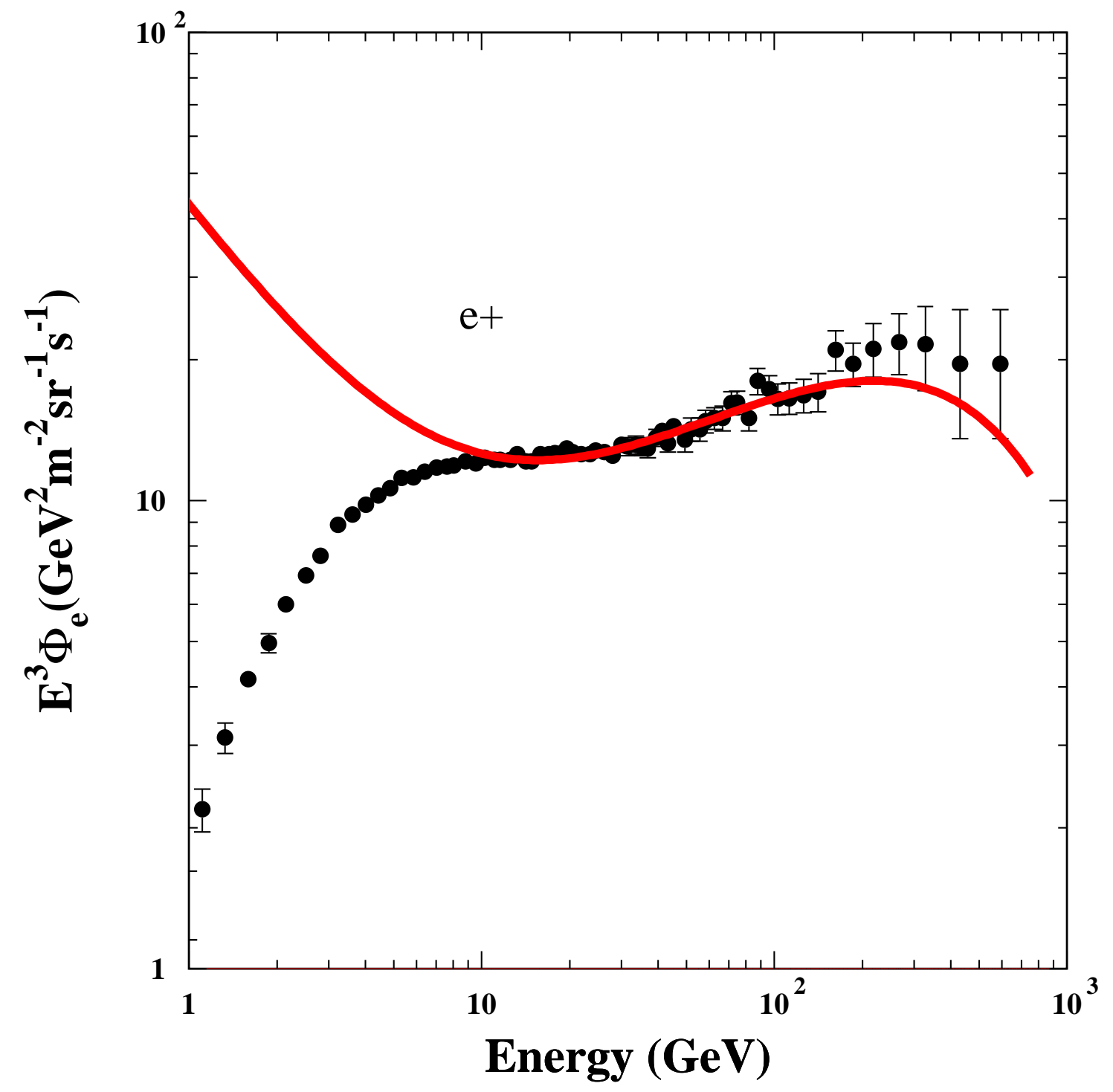

Fig. 6. - Comparison between the AMS minimal model (Accardo et al. 2014) best fit to the $\mathrm{e}^{+}$CR flux, Eq.(13), and the $\mathrm{e}^{+}$CR flux measured with AMS02 (Aguilar et al. 2014). 


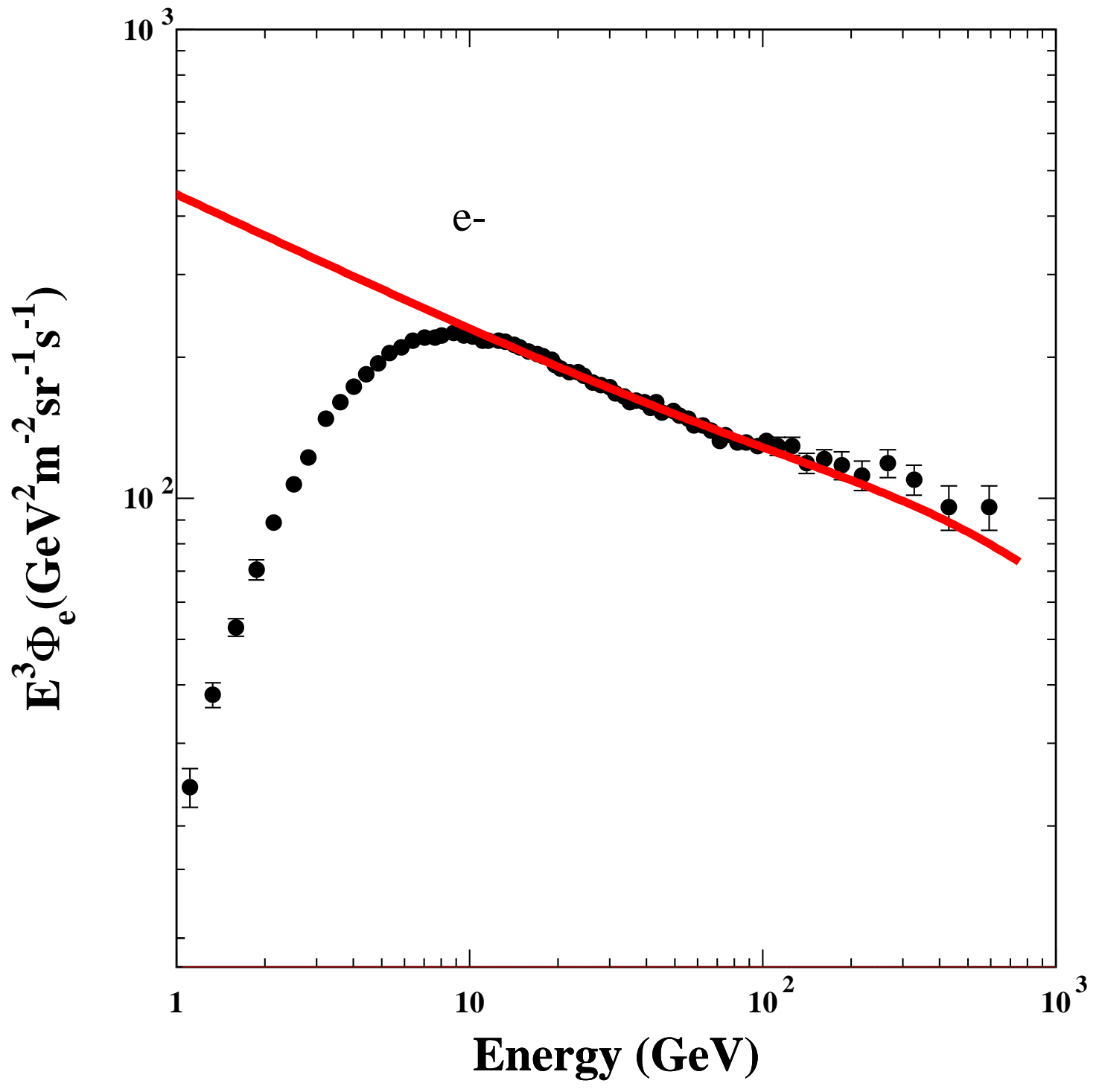

Fig. 7.- Comparison between the 'AMS minimal model' (Accardo et al. 2014) best fit to the $\mathrm{e}^{-}$CR flux, Eq.(14), and the $\mathrm{e}^{-}$CR flux measured with AMS02 (Aguilar et al. 2014). 


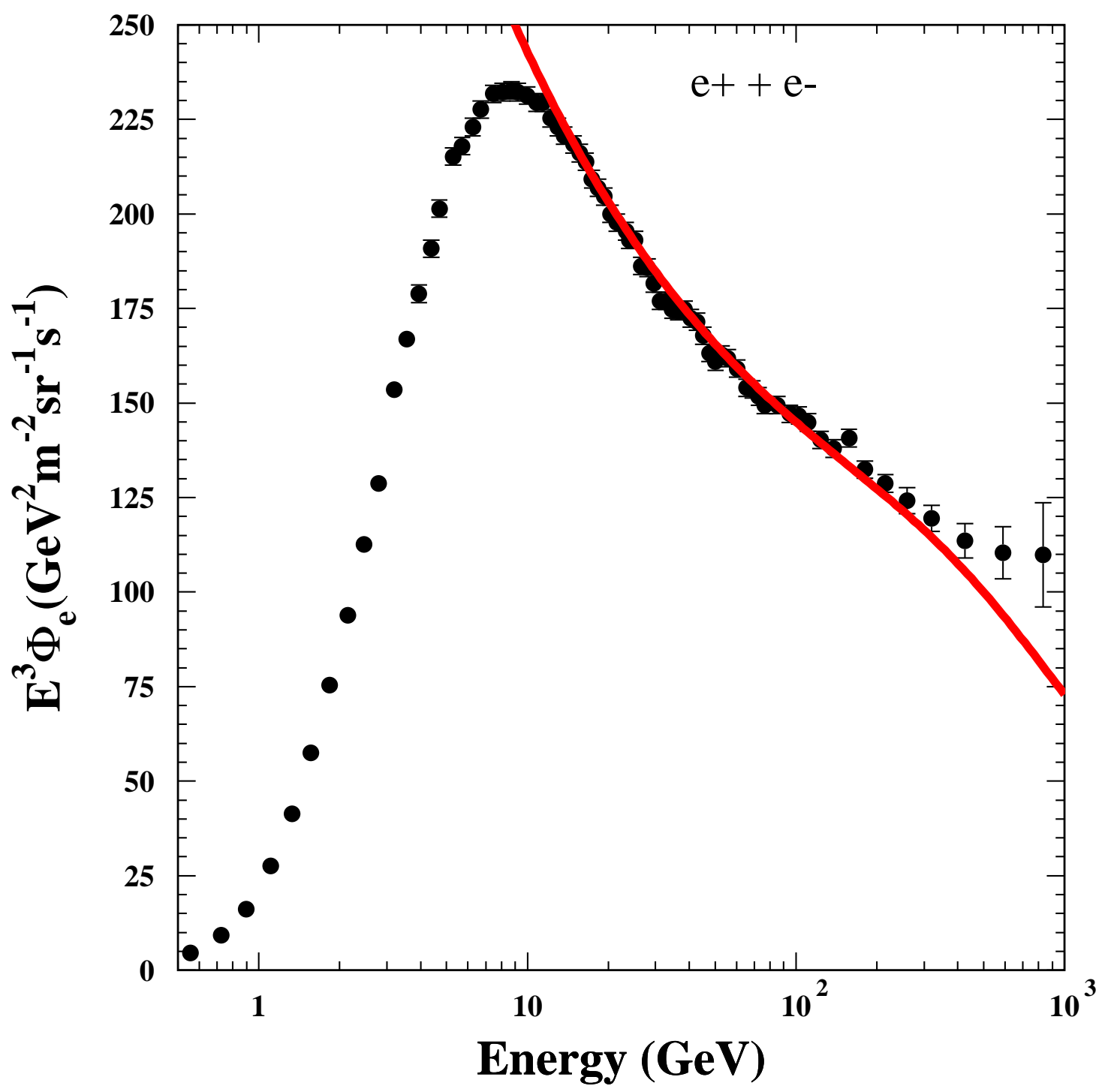

Fig. 8.- Comparison between the combined $\mathrm{e}^{ \pm}$CR flux measured with AMS02 near Earth (Aguilar et al. 2014) and the flux obtained from the best fits of the 'AMS minimal model' (Accardo et al. 2014) to their measured $\mathrm{e}^{+}$and $\mathrm{e}^{-}$fluxes. 


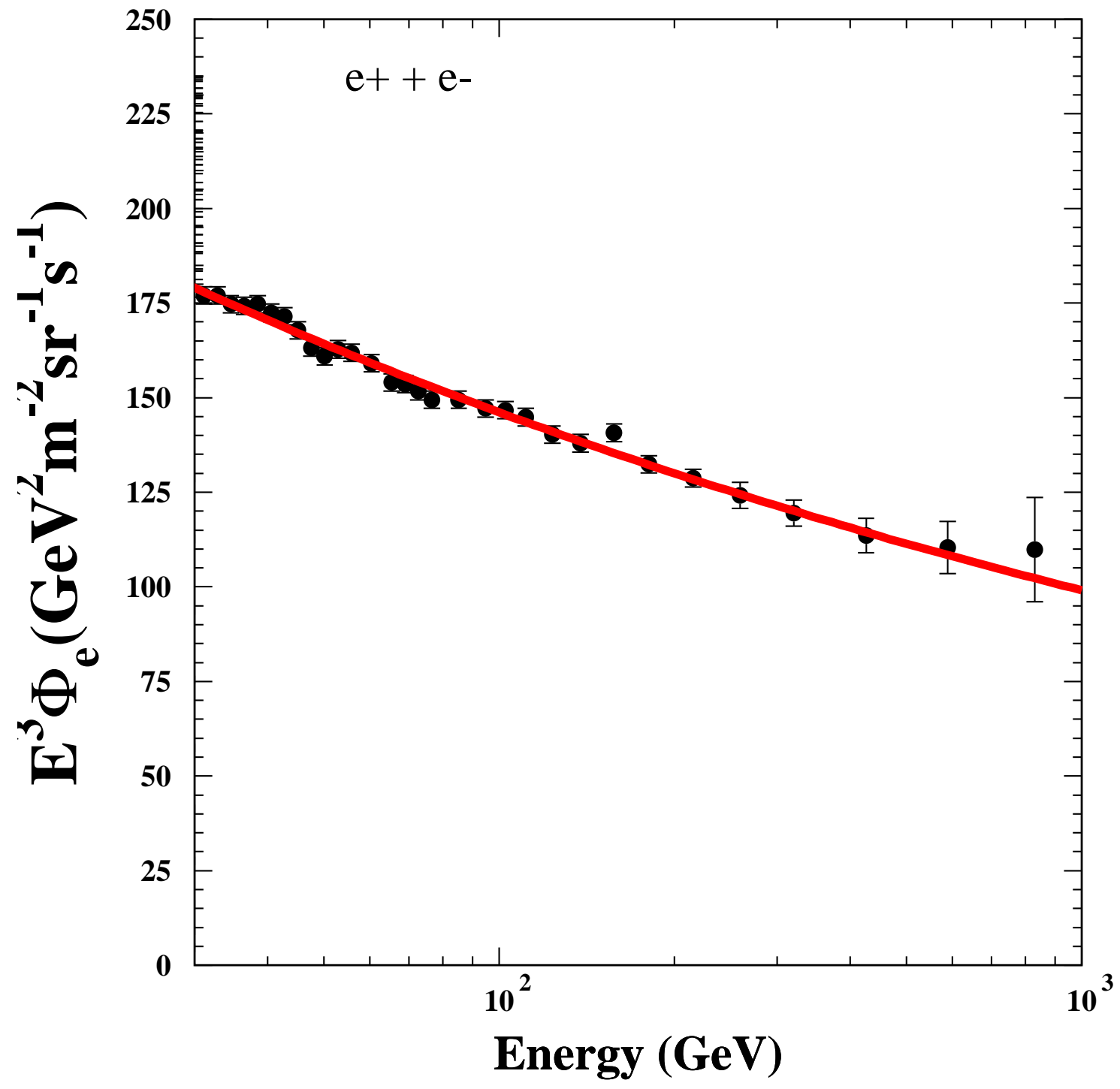

Fig. 9.- A power-law $\mathrm{A} \mathrm{E}^{-3.17}$ fit to the combined high energy $\mathrm{e}^{ \pm}$flux between $30-1000$ GeV measured near Earth with AMS02 (Aguilar et al. 2014). 


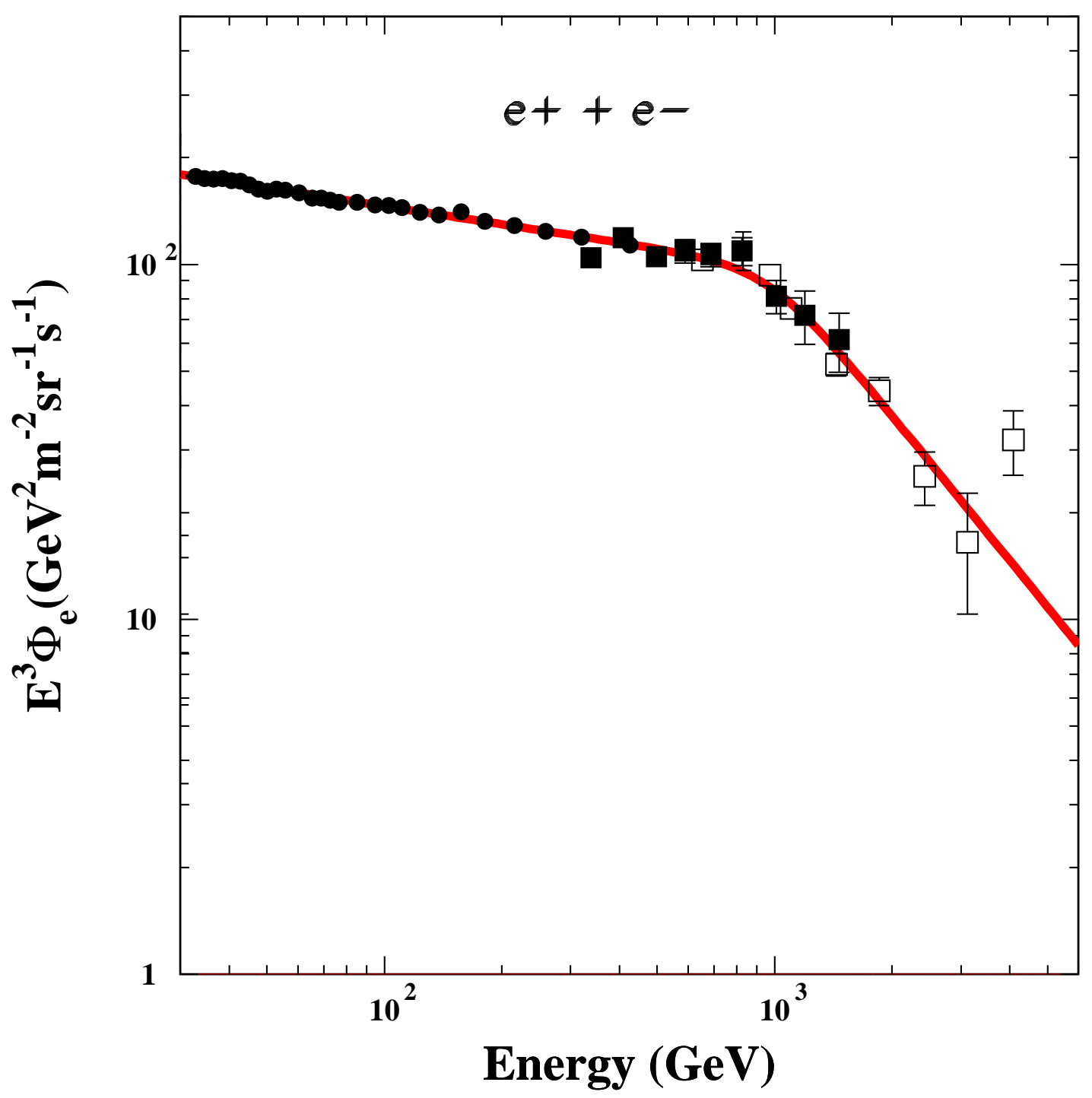

Fig. 10.- A cutoff power-law fit to the combined high energy $\mathrm{e}^{ \pm}$flux measured near Earth with AMS (full circles, Aguilar et al. 2014) and with H.E.S.S (squares, Aharonian et al. 2008,2009). The normalization of the H.E.S.S data was adjusted within their estimated systematic error to match the more precise AMS02 data below TeV (Aguilar et al. 2014). 